\title{
Oribates vecteurs \\ de Moniezia expansa (RUDOLPHI, 1810) du mouton dans la région de Fort-Lamy
}

\author{
par GRABER (M.) ef GRUVEL (J.) \\ (avec la collaboration technique de Mesdames BROCK et BRUNET)
}

\begin{abstract}
RÉSUMÉ
Les auteurs, après avoir donné quelques renseignements sur l'élevage de certaines espèces d'Oribates au laboratoire, signalent que, dans la région de Fort-Lamy Unguizetes reticulatus (WALLWORK, 1965), Scheloribates fimbriatus Thor africanus (WALLWORK, 1964) ef Scheloribotes perforatus (WALLWORK, 1964) sont susceptibles de transmettre à des moutons préalablement déparasités I'un des Cestodes les plus fréquents en Afrique centrale : Moniezia exponsa (RUDOLPHI, 1810).
\end{abstract}

La Monieziose ovine est une affection fort répandue de par le monde. Au Tchad, elle touche plus de 17 p. 100 du troupeau (GRABER, 1965 et 1968). Elle est due d la présence dans l'intestin des animaux atteints de deux Cestodes appartenant à la famille des Anoplocephalidae : Moniezio benedeni (MONIEZ, 1879) et Moniezio exponsa (RUDOLPHI, 1810) dont le cycle évolutif a été découvert en 1937 par STUNKARD aux U. S. A.

Le passage de mouton à mouton se fait par l'intermédiaire d'Acariens Oribates qui vivent normalement dans les sols ou sur les herbes. Des enquêtes effectuées çà et là en Europe, en Amérique et en Asie ont permis de mettre en évidence plus de 40 espèces différentes susceptibles de transmettre le parasite (MOREL, 1953 ; GRABER et GRUVEL, 1964 ; EUZEBY, 1966).

En Afrique, le laboratoire de Farcha a entrepris, dès 1962, des recherches d'Acarologie appliquée concernant plus particulièrement la biologie d'un Thysanosominae du mouton, Stilesia globipunctato (GRABER et GRUVEL, 1964 et 1967).

Parallèlement, Moniezia expanso n'était pas négligé et des études récentes ont abouti à un certain nombre de résultats qui font l'objet du présent travail.

\section{MATÉRIEL ET MÉTHODE}

10 Les vecteurs.

Les recherches ont porté sur les espèces suivantes :

- Oppia sp.

- Scheloribates perforatus (WALLWORK, 1964).

- Scheloribates fimbriatus Thor africonus (WALLWORK, 1964).

- Schelaribates parvus Van Pletzen conglobotus (WALLWORK, 1964).

- Unguizetes reticulatus (WALLWORK, 1965).

- Golumno baloghi (WALLWORK, 1965).

\subsection{Infestation naturelle.}

Unguizetes, Galumna et Oppia sp ont été récoltés. en dehors de la concession du laboratoire sur le pourtour d'une mare temporaire de saison des pluies. Située à une dizaine de kılomètres de 
Farcha, elle est fréquentée par d'importants troupeaux de moutons porteurs de Moniezia.

La terre suspecte de renfermer les Oribates est prélevée à des profondeurs variables. Elle est placée dans des seaux remplis d'eau. Le «surnageant» (débris végétaux-Oribates, etc.) est déposé dans une tolle de coton soigneusement formée et exposé durant $24 \mathrm{~h}$ à l'action d'un ventilateur de plafond. Après séchage, le contenu du torchon est tamisé et examiné sous la loupe. Les Oribates présents sont triés et classés par espèces.

\subsection{Infestation expérimentale.}

\subsubsection{Mode d'élevage des Oribates.}

Scheloribates perforatus, Scheloribates fimbriatus africanus et Scheloribates parvus conglobatus provenaient d'élevages réalisés au laboratoire.

a) En milieu naturel.

Au début, des bacs métallıques enterrés dans le sol ont été utilisés, selon une technique décrite précédemment (GRUVEL et GRABER, 1964). Malheureusement, ils demandent une survellance constante, car les Acariens ont tendance à s'échapper.

D'autres, au contraire, remontent le long des parois de la cuve, venant de l'extérieur, si bien qu'au bout de quelques mois la population est absolument différente de celle que l'on voulait obtenir à l'orıgine. Cette méthode a donc été provisoirement abandonnée.

b) In vitro.

D'autres procédés ont été expérımentés au laboratoire.

- Les Oribates sont mis dans des boîtes de Pétrı contenant de la terre, préalablement stérıIssée au four Pasteur et une rondelle de pomme de terre. Cette façon d'opérer présente des inconvénients : les boîtes de Pétri ne sont pas hermétiques et 11 existe souvent entre le rebord et le couvercle un interstice par lequel les Acoriens risquent de furr. Pour pallier cette difficulté, les récipients ont été disposés dans des plateaux remplis d'eau. Les Oribates, libres, sont facilement récupérés, mais ce système est long et immobilise du personnel. En outre, en saison des pluies, des souillures apparaissent : la pomme de terre devient gluante et les Acariens, pris dans une masse gélatineuse, meurent.

- Aussi, les boîtes de Pétrı ont-elles été rem- placées par des boîtes de Roux, plus vastes, remplies de la même manière, et dont l'ouverture est bouchée par du coton cardé, ce qui évite les évasions.

- Avant d'infester les Scheloribates, on attend l'éclosion de la deuxième génération, car la première est souvent parasitée, ce qui peut amener des confusions lors de l'infestation expérimentale des moutons.

- Pour des Oribates mis en élevage durant I'hivernage, la deuxième génération apparaît en général vers la mi-décembre. Les individus qui la constituent sont répartis dans de nouvelles boîtes de Roux.

\subsubsection{Infestation.}

Les Cestodes servant à l'infestation sont prélevés dans l'intestin d'ovins sacrifiés à l'abattoir. Seuls les derniers anneaux de la chaîne où se trouvent les cufs mûrs sont récoltés. Un fragment est mis de côté pour détermination, après coloration au carmin chlorhydrique.

Le reste est broyé dans un mortier avec un peu de terre sèche et le produit ainsi obtenu est passé, à l'aide d'un fin pinceau, à la surface de la rondelle de pomme de terre où les Scheloribates sont toujours nombreux.

Pour réussir une bonne infestation, il faut renouveler l'opération au moins 6 fois.

Cette méthode, dans le cas des Scheloribates, a donné d'excellents résultats.

Cependant elle n'est pas parfaite car la rondelle de pomme de terre ainsi traitée est capable de pourrir rapidement et il est recommandé de la remplacer au bout de $48 \mathrm{~h}$.

\section{$2^{\circ}$ Les animaux d'expérience.}

39 moutons, originaires des environs de FortLamy ont été soumıs à des infestations expérimentales. Compte tenu de l'incidence élevée du Téniasis dans la région, les ovins ont subi un traitement préalable à la Niclosamide Bayer*.

La dose utılısée est de $150 \mathrm{mg} / \mathrm{kg}$, ce qui assure l'expulsion des Moniezia, des Avitellino et d'un grand nombre de Stilesia globipunctota (GRABER, 1969).

Comme lors des essais précédents (GRABER ef GRUVEL 1964 ef 1967), les animaux ainsi

\footnotetext{
* Yomesan ou Mansonil.
} 


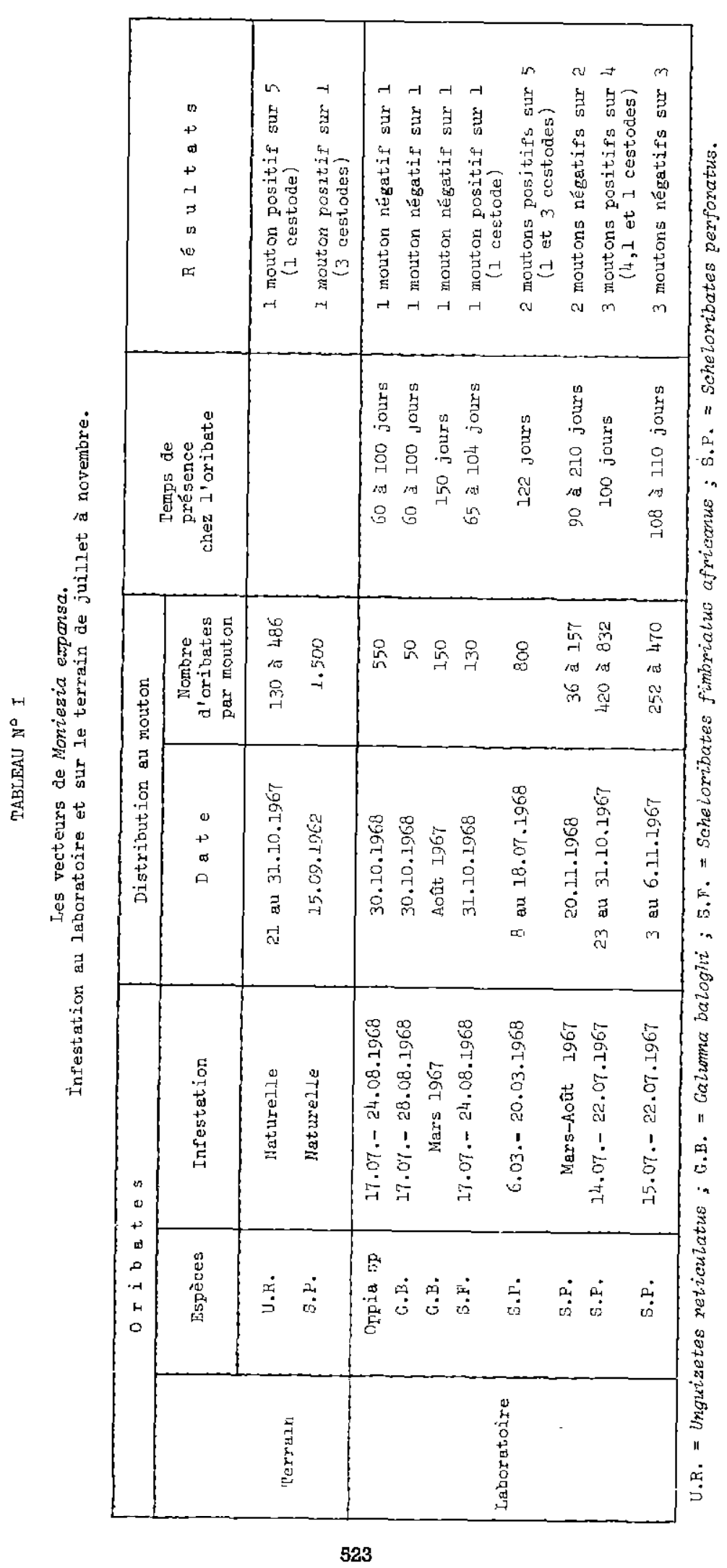


préparés ont été mis dans des stalles cimentées ef nourris, dès leur arrivée, avec du fourrage vert coupé sur les bords du fleuve Charı dans des zones où if n'y a pas d'Artıodactyles domestiques ou sauvages susceptibles d'héberger Moniezia expansa. Les herbes ont été rigoureusement contrôlées tous les jours. Des branches d'arbres ont également été ajoutées à la ration.

Les Acariens déterminés et comptés sont administrés au mouton dans de l'eau, «à la bouteille ».

\section{RÉSULTATS}

Le tableau $n^{0} 1$ fait le bilan des résultats obtenus depuis 1962.

Quelques points méritent de retenir plus particulièrement l'attention.

10 Moniezia exponso, au Tchad, a pu être transmis au mouton 6 fois à partir de Scheloribotes perforatus, une fois à partir de Scheloribotes fimbriotus africanus et une fois à partir d'Unguizetes reticulatus.

II n'y a donc pas à proprement parler, de spécificité de vecteurs. Plusieurs espèces peuvent jouer également le rôle. Le même phénomène a été constaté dans d'autres pays, en Bulgarie (BANKOV, 1965) et en Hongrie où des Cysticercoldes de Moniezia ont été retrouvés chez 6 acariens différents (KASSAl et MAHUNKA, 1965). Ces auteurs pensent que tout Oribate dont la taille est supérieure à $300-400 \mu$ doit être considéré comme un hôte possible, ce qui paraît vraisemblable, dans la région de Fort-Lamy, quand on considère les dimensions des Acariens les plus fréquemment rencontrés (tableau no 2).

$2^{\circ}$ Le pourcentage d'Oribates infestés est en général peu élevé, tant dans les conditions naturelles que dans les conditions expérimentales:

- 0,16 p. 1.000 avec Scheloribotes fimbriatus africanus.

- 0,93 p. 1.000 avec Scheloribates perforatus.

- 0,88 p. 1.000 avec Unguizetes reticulatus.

Aussi la mise en évidence des Cysticercoïdes, après dissection des vecteurs, est-elle difficile, voire impossible.

Les taux d'infestation semblent nettement inférieurs à ce qu'ils sont dans d'autres pays, notamment en Amérique et en Europe où, sı l'on s'en tient aux chiffres de MOREL (1953), ils dépassent en moyenne 15 p. 1.000 . On observe d'ailleurs d'importantes variations qui dépendent de l'implantation des élevages ovins, des Oribates en cause et du climat.

$3^{\circ}$ Le nombre de Moniezıa recueillis à l'autopsie des moutons d'expérience est faible : un par animal, au maximum trois.

Les premiers œufs apparaissent dans les selles entre 45 et 55 jours, ce qui confirme des observations précédentes (GRABER et SERVICE, 1964).

Quand le Cestode est unique, le rythme de croissance est le suivant :

TABLEAU N $\mathrm{N}^{\circ}$ II

Les vecteurs de Monieaia exponsa - Infestation eu laboratoire de décembre à juin.

\begin{tabular}{|c|c|c|c|c|c|}
\hline \multicolumn{2}{|r|}{ Orabates } & \multicolumn{2}{|c|}{ Distribution au mouton } & \multirow{2}{*}{$\begin{array}{c}\text { Temps de } \\
\text { présence } \\
\text { chez l'oribate }\end{array}$} & \multirow[b]{2}{*}{ Résultats } \\
\hline Espèces & Infestation & Date & $\begin{array}{c}\text { Nombre } \\
\text { a'oribetes } \\
\text { par mouton }\end{array}$ & & \\
\hline S.P.C. & $27.07-20.08 .1968$ & 30 et 31.12 .1968 & 75 & 100 è 125 jours & 1 routon négatif sur 1 \\
\hline S.F. & 11 au 17.11 .1966 & 3 au 8.05 .1967 & 1.000 & 175 jours & 3 moutons négetifs sur 3 \\
\hline S.F. & $21.10-21.11 .1966$ & $30.05-3.06 .1967$ & 1.500 & 165 ¿̀ 220 jours & 2 moutons négatizs sur 2 \\
\hline $\begin{array}{l}\text { Cribates } \\
\text { divers }\end{array}$ & $21.10-21.11 .1966$ & 5.06 .1967 & 1.500 & 195 à 225 jours & 2 moutons négetizs sur 2 \\
\hline S.P. & $29.07-18.09 .1967$ & 4 et 5.01 .1968 & 95 et 290 & 110 à 185 jours & 2 moutons négatifs sur 2 \\
\hline S.P. & 18 au 25.02 .1967 & 27.06 .1967 & 350 & 120 玉̀ 130 jours & I mouton négatif sur = \\
\hline S.F. & $25.07-27.08 .1968$ & 23 घu 27.12.1968 & 710 & 120 ̇े 150 jours & 4 moutons négatifs sur 2 \\
\hline
\end{tabular}

S.P.C. = Scheloribates parvus congtobatus; S.F. = Soneloribates fimbriatus africanus;

S.P. = Seneioribates perforatus. 
-20 jours : $8,5 \mathrm{~cm}$,

- 34 à 39 jours : 70 à $80 \mathrm{~cm}$,

- 45 jours : $1,50 \mathrm{~m}$,

- 50 jours : $1,91 \mathrm{~m}$,

- 56 jours : 2,30 à $3,49 \mathrm{~m}$.

Parfois elle est refardée et, à 34-36 jours, la longueur du parasite est moindre $(30 \mathrm{o} 60 \mathrm{~cm}$ ).

Lorsqu'il existe plusieurs Moniezia, ils sont toujours, à âge égal, de plus petite taılle (de 25 à $30 \mathrm{~cm}$ à 35 jours pour 3 Cestodes et de 15 à $60 \mathrm{~cm}$ à 40 jours pour 4 Cestodes).

40 Il faut tenir compie également de la saison: l'infestation des moutons à partir d'Oribates porteurs de Cysticercoïdes est, dans 44 p. 100 des cas, positive de juillet à novembre. Cette époque correspond à la saison des pluies et au début de la saison sèche.

Par contre, de décembre à juın, aucun passage n'a pu être réalısé.

Les observations faites tant au laboratoire que sur le terrain coïncident étroıtement : le Téniasis ovin, dans la région de Fort-Lamy, est essentiellement une affection d'hivernage qui débute en juillet pour se terminer en janvier de l'année suivante (GRABER et SERVICE, 1964).

Les trois vecteurs actuellement connus dont la biologie est différente (GRUVEL et GRABER, 1967) se relaient pour assurer, à la période la plus favorable, la transmission de la maladie.

50 Après infestation expérimentale, le nombre de moutons hébergeant Moniezıa exponsa est de 20,5 p. 100 , ce qui est légèrement supérieur à la moyenne nationale (tableau $n^{\circ} 3$ ), mais inférieur de 5 p. 100 au pourcentage obtenu dans la région de Fort-Lamy. L'écart ne paraît donc pas très sensible.

\section{CONCLUSIONS}

Plusıeurs espèces d'Oribates sont capables, tant dans les conditions naturelles qu'expéri-

TABLEAU $N^{\circ}$ III

Dimensions des espèces dominantes dans la région de Fort-Lamy (d'après Wallwork 1964 et 1965)

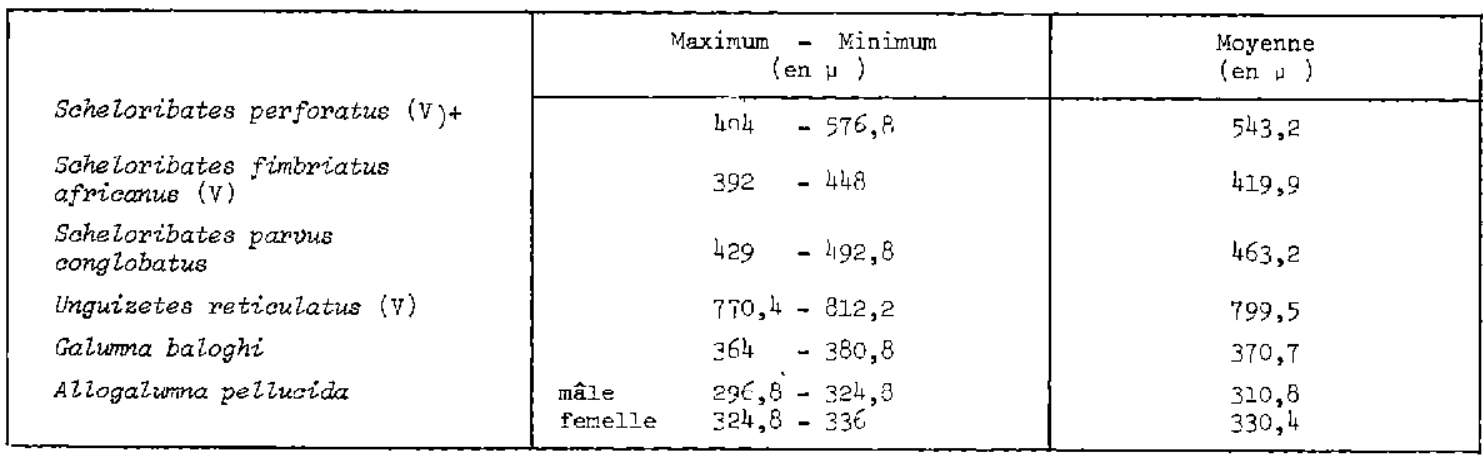

$+(v)=$ vecteurs de Moniezia exponsa.

TABLEAU $x^{\circ}$ IV

Nombre de moutons porteurs de Moniezta expansa

\begin{tabular}{|c|c|c|c|}
\hline \multirow{2}{*}{$\begin{array}{c}\text { Totelité du Tchad } \\
\text { (1954 - 1969) }\end{array}$} & $\begin{array}{c}\text { Nombre d'animaux } \\
\text { examinés }\end{array}$ & $\begin{array}{c}\text { Ncmcre a'erimaux } \\
\text { porteurs de } \\
\text { [foniezia exponsa }\end{array}$ & $\begin{array}{c}\text { Pourcentage } \\
\text { d'infestation }\end{array}$ \\
\cline { 2 - 4 } & 5.567 & 980 & $17,6 \mathrm{p} .100$ \\
\hline Fort-Lamy & 3.062 & 783 & $25,5 \mathrm{p} .100$ \\
\hline $\begin{array}{l}\text { Infestations } \\
\text { expérimentales }\end{array}$ & 39 & 8 & $20,5 \mathrm{p} .100$ \\
\hline
\end{tabular}


mentales, de transmettre au mouton Moniezıa exponsa. Dans la région de Fort-Lamy, ce sont Unguizetes reticulatus (WALLWORK, 1965), Scheloribotes fimbriatus Thor africanus (WALLWORK, 1964 ) et Scheloribates perforatus (WALLWORK, 1964).

Le nombre d'Acariens porteurs de Cysticercoldes est peu élevé et ne semble pas dépasser
1 p. 1.000. II en est de même pour les Cestodes recueillıs dans l'intestin à l'autopsie.

Au laboratoire, l'époque la plus favorable à l'infestation des animaux d'expérience va de juillet à novembre : le Téniasis ovin est donc une affection de saison des pluies ef de début de saison sèche, ce qui confirme les résultats des enquêtes helminthologiques prélimınaires.

\title{
SUMMARY \\ Oribates as vectors of Moniezia exponso (RUDOLPHI, 1810) \\ of sheep in the Fort-Lamy area
}

\begin{abstract}
At once, the authors give some informations about the breeding of some Oribates species in the laboratory. Then, they indicate that in the Fort-Lamy area, Unguizete reticulatus (WALLWORK, 1965), Scheloribates fimbriatus Thor africanus (WALLWORK, 1964) and Scheloribatus perforatus (WALLWORK, 1964) can propagate to previausly treated sheep one of the more frequent cestode of Central Africa Montezia expansa (RUDOLPHI, 1810).
\end{abstract}

\section{RESUMEN}

Oribatos vectores de Moniezia expanso (RUDOLPHI, 1810) de la oveja en la region de Fort-Lamy

\begin{abstract}
Los autores dan algunas informaciones sobre la crianza de clertas especies de Oribatos en el Laboratorio. Luego, señalan que, en la región de Fort-Lamy, Unguizetes reticulatus (WALLWORK, 1965), 5cheloribates fimbriatus Thor africanus (WALLWORK, 1964) y Scheloribates perforotus (WALLWORK, 1964) pueden transmitr a las ovejas previamente deparasitadas uno de los céstodos más frecuentes en Africa central : Moniezio exponsa (RUDOLPHI, 1810).
\end{abstract}

\section{BIBLIOGRAPHIE}

EUZEBY (J.). - Les maladies vermineuses des animaux domestiques ef leurs incidences sur la pathologie humaine. T. II. Maladies dues aux Plathelminthes. Fasc. I. Cestodoses. Vigot Frères, Paris, 1966, 663 p.

GRABER (M.) et GRUVEL (J.). - Note préliminaire concernant la transmission de Stilesia globipunctafo (RIVOLTA, 1874) du mouton par divers Acariens Oribates. Rev. Elev. Méd. Vét. Pays trop., 1964, 17, 3 , 467-76.

GRABER (M.) et SERVICE (J.). - Le Téniasis des bovins ei des ovins de la République du Tchad. Quelques données épidémiologiques concernant les zones sahéliennes. Rev. Elev. Méd. Vét. Pays trop., 1964, 17, 3, 491-521.

GRABER (M.). - Helminthes et Helminthiases faisant obstacle à l'amélioration de la production ovine en République du Tchad. Monographie 1965, $158 \mathrm{p}$.

GRABER (M.) ef GRUVEL (J.). - Les vecteurs de Stilesia globipunctata (RIVOLTA, 1874) du mouton. Rev. Elev. Méd. Vét. Pays trop., $1967,20,2,261-71$.

GRABER (M.). - Helminthes ef Helminthiases. Bilan d'activité. Rap. Ann., 1967. Laboratoire de Farcha, t. III, 1968, 192 p. 
GRABER (M.). - A propos du pouvoir anthelminthique du N-(-2'-Chloro-4' nitrophenyl)-5 Chlorosalicylamide. Rev. Elev. Méd. Vét. Poys trop., 1969 (à paraître).

GRUVEL (J.) et GRABER (M.). - Récolte et mise en élevage d'Acariens oribates dans les conditions tchadiennes. Rev. Elev. Méd. Vét. Pays trop., 1964, 17, 3, 575-81.

GRUVEL (J.) et GRABER (M.). - Premières remarques écologiques concernont quelques espèces d'Oribates au Tchad. Rev. Elev. Méd. Vét. Pays trop., 1967, 20, 3, 457-73.

KASSAI (T.) ef MAHUNKA (S.). - Studies on tapeworms in rumınants II. Oribatides as intermediate hosts of Moniezia species. Acta Vet. Hung., 1965, 15, 2, 227-49.

MOREL ( $P$.). - Les Cestodes du mouton. Thèse vétérinaire, Paris, 1953, $93 \mathrm{p}$.

SPASSKI (A. A.) - Essentials of Cestodology. Vol. I. Anoplocephalate tapeworms of domestic and wild animals. Moscov, 1951.

STUNKARD (H. IU). - The life-cycle of Moniezio expansa. Science, 1937, 86, 312.

WALLWORK (Y. A.). - Some oribatel (Acari : Cryptostigmata) from Tchad (Ist. Series). Rev. zool. Bot. Afr., 1964, 70, 3/4, 353-85. WALLWORK (J. A.). - Some Oribatei (Acari : Cryptostigmato) from Tchad (2nd Series). Rev. zool. Bot. Afr., 1965, 72, 1/2, 83-108. 